\title{
Identification of a potent botulinum neurotoxin A protease inhibitor using in situ lead identification chemistry
}

\author{
Grant E. Boldt, Jack P. Kennedy, and Kim D Janda* \\ Departments of Chemistry and Immunology, The Skaggs Institute for Chemical Biology, and Worm \\ Institute of Research and Medicine (WIRM), The Scripps Research Institute, 10550 North Torrey \\ Pines Road, La Jolla, CA 92037, USA.
}

\section{Abstract

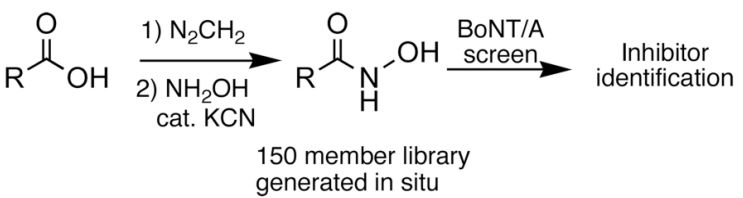

Botulinum neurotoxins (BoNTs), etiological agents of the deadly food poisoning disease botulism, are the most toxic proteins currently known. By using in situ lead identification chemistry we have uncovered the first class of inhibitors that display nanomolar potency. From a $15 \mu \mathrm{M}$ lead compound, structure activity relationship studies were performed granting the most potent BoNT/A inhibitor reported to date that displays an inhibition constant of $300 \mathrm{nM}$.

Botulinum neurotoxin (BoNT), an agent responsible for the deadly food poisoning disease botulism and a dreaded biological weapon, is one of the most toxic proteins currently known ( 100 billion times more toxic than cyanide). ${ }^{1}$ Clostridium botulinum is classified into seven strains $(A-G)$ each of which can cause flaccid muscle paralysis and subsequent death by blocking the release of a neurotransmitter, acetylcholine, at neuromuscular junctions. ${ }^{2}$ Structurally, BoNT consists of three functional domains; catalytic, translocation, and binding; BoNT toxicity results from the catalytic activity of its light chain, a Zn(II) endopeptidase.

The catalytic domain of BoNT is a compact globule consisting of a mixture of $\alpha$-helices, $\beta$ sheets and strands with a gorge-like zinc containing metalloprotease active site (15-20 $\mathrm{A}$ deep depending on serotype). ${ }^{3}$ The metalloprotease activity is responsible for BoNT's neurotoxicity through the hydrolytic cleavage of one of three SNARE (soluble NSF-attachment protein receptor) proteins that are involved in neuronal synaptic vesicle function. Moreover, the hydrolytic cleavage sites of these SNARE proteins (SNAP-25, VAMP, Sb-1) differ across the BoNT serotypes; however, any degradation of these SNARE proteins disables the exocytosis of acetylcholine, resulting in paralysis and potentially death. ${ }^{2}$ Current therapy for BoNT intoxication involves "passive immunization" with equine antitoxin. ${ }^{4}$ Unfortunately, treatment must start shortly after intoxication, and several safety concerns exist ${ }^{2}$ over the use of antitoxins in the general population. ${ }^{5}$ Therefore, inhibition of the catalytic light chain protease with a small molecule inhibitor may provide an attractive approach to counter the effects of botulism poisoning.

BoNT serotype A (BoNT/A) is the most toxic form of BoNT's and is considered the most threatening for biological attacks due to a prolonged half-life in vivo and ease of its production. 
${ }^{4}$ While there are reports of success treating BoNT/A toxicity with multiple monoclonal antibodies ${ }^{6}$ as antitoxins this is of limited therapeutic utility since the antibodies must be administered prior to, or shortly after, toxin exposure ( $<12 \mathrm{hrs})$.

Presently, there are only modest small molecule, non-peptidic, protease inhibitors for BoNT/ A with $\mathrm{IC}_{50}$ values in the range of $>20 \mu \mathrm{M} .{ }^{7}$ We established a high-throughput screen for the identification of inhibitors of BoNT/A LC protease. ${ }^{8}$ Using this screen we have analyzed a library of hydroxamate-based compounds generated using in situ chemistry to reveal the lead structure 1, (Figure 1). Herein, we report upon the synthesis and structure activity relationship studies of these BoNT/A inhibitors.

Recently, a convenient method for the preparation of hydroxamates from readily available esters was reported. ${ }^{9}$ In this general procedure a diverse array of acids can easily be converted to hydroxamates with hydoxylamine in the presence of a catalytic amount of potassium cyanide. In addition, several reports have been disclosed for the preparation of hydroxamates; ${ }^{10}$ however, these procedures have strict substrate requirements.

With these thoughts in mind, we set out to generate a library of diverse hydroxamates from a readily available carboxylic acid library. Namely, 150 carboxylic acids purchased from Aldrich Chemical Company were randomly chosen and converted to their ester by treatment with diazomethane (Scheme 1). After removal of the solvent, reactions were subjected to a mixture of THF:MeOH:HONH ${ }_{2}$ 1:1:2 with a catalytic amount of $\mathrm{KCN}$ overnight. Again, the solvent was removed and the crude products were reconstituted with DMSO to prepare stock solutions of a final concentration of $10 \mathrm{mM}$ for screening. To check the quality of the library 30 compounds were randomly selected and analyzed by ES-MS, in all cases the expected masses corresponding to the products were found (data not shown).

Using a high-throughput screen, ${ }^{8}$ namely, a 17 amino acid substrate fluorescence resonance energy transfer (FRET) assay developed in our laboratory, the library of hydroxamates were analyzed at a concentration of $50 \mu \mathrm{M}$. Any inhibitors found to display $50 \%$ inhibition were considered "hits" and evaluated further. From the initial screen five compounds were found to give $50 \%$ or more inhibition in the FRET-based assay. These compounds were resynthesized, purified and validated using the FRET-based assay. Of the five lead compounds, only compound $\mathbf{1}$ showed potent activity; para-chloro-cinnamic hydroxamte (1) displayed an $\mathrm{IC}_{50}$ of $15 \mu \mathrm{M}$, and thus this simple high-throughput screen uncovered an interesting lead for further development.

To refine this positive lead we synthesized a series of 12 compounds so as to further explore the structure activity relationship of compound $\mathbf{1}$. However, the method outlined in Scheme 1 was found to be inefficient for the isolation of the desired compounds due to purification difficulties. Therefore, we sought out a more convenient method for the expeditious generation and purification of the hydroxamate compounds. Solid-phase organic synthesis (SPOS) lends itself to these requirements nicely due to reactions proceeding in high yield, and purification being significantly simplified. Several groups have demonstrated successful hydroxamate syntheses using solid phase routes ${ }^{11}$ and hydroxyamine resins. ${ }^{12}$ However, we found these procedures to be insufficient for our purposes. 2-Chlorotrityl resin was purchased from Chemical and Biopharmaceutical Laboratories of Patras S. A., (CBL), and used in the synthesis of hydroxamate inhibitors as outlined in Scheme 2. It is important to note that several resins were explored using this methodology, however, the CBL was the only resin found to give satisfactory results. This resin was treated with $N$-hydroxyphthalimide for $2 \mathrm{~d}$ in DMF at room temperature for the quantitative conversion to $\mathbf{6}$. The phthalimide was successfully removed with exposure to anhydrous hydrazine to give the hydroxylamine resin $\mathbf{7}$, ready for the coupling of a variety of acids. Next, the desired carboxylic acids were coupled to the resin 7 using 
standard diisopropylcarbodiimide, and 6-chlorohydroxybenzotriazole protocols. The desired hydroxamates were recovered by treatment of the resin with 5\% TFA and removal of the solvent.

These compounds were further individually purified via silica gel, then reconstituted in DMSO for evaluation of BoNT/A LC inhibition.

In order to investigate the structure activity relationship of compound $\mathbf{1}$ we first synthesized a group of para substituted cinnamic hydroxamates. Unexpectedly, the chloro substitution in the para position appears to be highly conserved as seen in Table 1 compounds 10-16. For example, when a bulky functionality such as a $t$-butyl, compund $\mathbf{1 4}$, is introduced to the para position, inhibition is completely lost. Moreover, inhibition is compromised when introducing electron withdrawing or donating groups (compounds 10-16). Thus, we next examined other possible chloro substitution patterns of the lead compound 1. Gratifyingly, when evaluating different chloro substitution patterns, (compounds 17 and 18), the orthopara cinnamic hydroxamate, 18, displayed an $\mathrm{IC}_{50}$ of $0.41 \mu \mathrm{M}$. Furthermore, it appears the trans olefin motif is also conserved as seen in compounds $\mathbf{1 9 , 2 0}$, and $\mathbf{2 1}$. It is important to note, the cis-olefin form of , $\mathbf{1}$, was not examined due to instability in solution. ${ }^{13}$ However, strikingly, when the trans olefin is substituted for an alkyne, 20, inhibition was attenuated by greater than 175 -fold. The reduced form of $\mathbf{1 8}$ to the alkane, compound $\mathbf{1 9}$, also reduced potency significantly.

To further kinetically characterize $\mathbf{1 8}$, we evaluated it in an assay system with the native SNAP-25 (141-206) substrate using HPLC for analysis. It is important to note, that the SNAP-25 (141-206) has no structural modification, thus the structural integrity of the molecule has not been compromised. Using this substrate, a $K_{\mathrm{i}}$ of $300 \mathrm{nM} \pm 12 \mathrm{nM}$ was found, and also displayed the expected competitive mode of inhibition (data not shown). Moreover, these findings demonstrate that a 10 contiguous carbon chain previously thought to be a strict requirement for BoNT/A LC inhibition ${ }^{14}$ is in fact not a prerequisite and small organic molecules can be used for possible drug leads.

In total, a simple in situ synthesis and screen was developed for the identification of nonpeptidic protease inhibitors of BoNT/A LC. The power of using this strategy was that no prior bias was placed on the selection of input acids, yet, a desirable lead was uncovered. We note that compound 18, Table 1, is the most potent protease inhibitor described to date for BoNT/ A and is currently being investigated in cell and mouse assays for anti-botulism effects and will be reported in due course.

\section{Supplementary Material}

Refer to Web version on PubMed Central for supplementary material.

\section{Acknowledgments}

This work was supported by the National Institute of Health BT010-04 and The Skaggs Institute for Chemical Biology.

\section{References}

1. Singh BR. Nature 2000;7:617-619.

2. Simpson LL. Annu. Rev. Pharmacol. Toxicol 2004;44:167-193. [PubMed: 14744243]

3. Lacy BD, Tepp W, Cohen AC, DasGupta BR, Stevens RC. Nat. Struct. Biol 1998;5:898-902. [PubMed: 9783750]

4. Hicks RP, Hartell MG, Nichols DA, Bhattacharjee AK, Von Hamont JE, Skillman DR. Curr. Med. Chem 2005;12:667-690. [PubMed: 15790305] 
5. Arnon SS, Schechter R, Inglesby TV, Henderson DA, Bartlett JG, Ascher MS, Eitzen E, Fine AD, Hauer J, Layton M, Lillibridge S, Osterholm MT, O'Toole T, Parker G, Perl TM, Russell PK, Swerdlow DL, Tonat K. JAMA 2001;285:1059-1070. [PubMed: 11209178]

6. (a) Amersdorfer P, Wong C, Smith T, Chen S, Deshpande S, Sheridan R, Marks JD. Vaccine 2002;20:1640-1648. [PubMed: 11858873] (b) Nowakowski A, Wang C, Powers DB, Amersdorfer P, Smith TJ, Montgomery VA, Sheridan R, Blake R, Smith LA, Marks JD. Proc. Natl. Acad. Sci. USA 2002;99:11346-11350. [PubMed: 12177434]

7. (a) Burnett JC, Schmidt JJ, Connor F, McGrath CF, Nguyen TL, Hermone AR, Panchal RG, Vennerstrom JL, Kodukula K, Zaharevitz DW, Gussio R, Bavari S. Bioorg. Med. Chem 2005;13:333341. [PubMed: 15598556] (b) Burnett JC, Schmidt JJ, Stafford RG, Panchal RG, Nguyen TL, Hermone AR, Vennerstrom JL, McGrath CF, Lane DJ, Sausville EA, Zaharevitz DW, Gussio R, Bavari S. Biochem. Biophys. Res. Commun 2003;310:84-93. [PubMed: 14511652]

8. Boldt GE, Kennedy JP, Hixon MS, McAllister LA, Barbieri JT, Tzipori S, Janda KD. J. Comb. Chem. 2006submitted

9. Ho CY, Strobel E, Ralbovsky J, Galemmo RA. J. Org. Chem 2005;70:4873-4875. [PubMed: 15932334]

10. (a) Burns CJ, Groneberg RD, Salvino JM, McGeehan G, Condon SM, Morris R, Morrissette M, Mathew R, Darnbrough S, Neuenschwander K, Scotese A, Djuric S, Ullrich J, Labaudiniere R. Angew. Chem., Int. Ed 1998;37:2848-2850. (b) Mori K, Koseki K. Tetrahedron 1988;44:6013-6020. (c) Spengler J, Burger K. Synthesis 1998;1:67-70.

11. (a) Dankwardt SM. Synlett 1998;7:761. (b) Dankwardt SM, Billedeau RJ, Lawley LK, Abbot SC, Martin RL, Chan CS, Van Wart HE, Walker KE. Bioorg. Med. Chem. Lett 2000;10:2513-2516. [PubMed: 11086718]

12. (a) Barlaam B, Koza P, Berriot J. Tetrahedron 1999;55:7221-7232. (b) Mellor SL, McGuire C, Chan WC. Tetrahedron Lett 1997;38:3311-3314. (c) Floyd CD, Lewis CN, Patel SR, Whittaker M.

Tetrahedron Lett 1996;37:8045-8048. (d) Ede NJ, James IW, Krywuth BM, Griffiths RM, Eagle SN, Gubbins B, Leitch JA, Sampson WR, Bray AM. Lett. Pep. Sci 1999;6:157-163. (e) Bauer U, Ho WB, Koskinen AMP. Tetrahedron Lett 1997:7233-7236.

13. Cis-2,4, dichloro-cinnamic acid was found to be unstable during coupling.

14. Park JG, Sill PC, Makiyi EF, Garcia-Sosa AT, Millard CB, Schmidt JJ, Pang YP. Bioorg. Med. Chem 2006;14:395-408. [PubMed: 16203152] 
<smiles>O=C(/C=C/c1ccc(Cl)cc1)NO</smiles>

Figure 1.

Structures of lead compounds identified from in situ screen. 


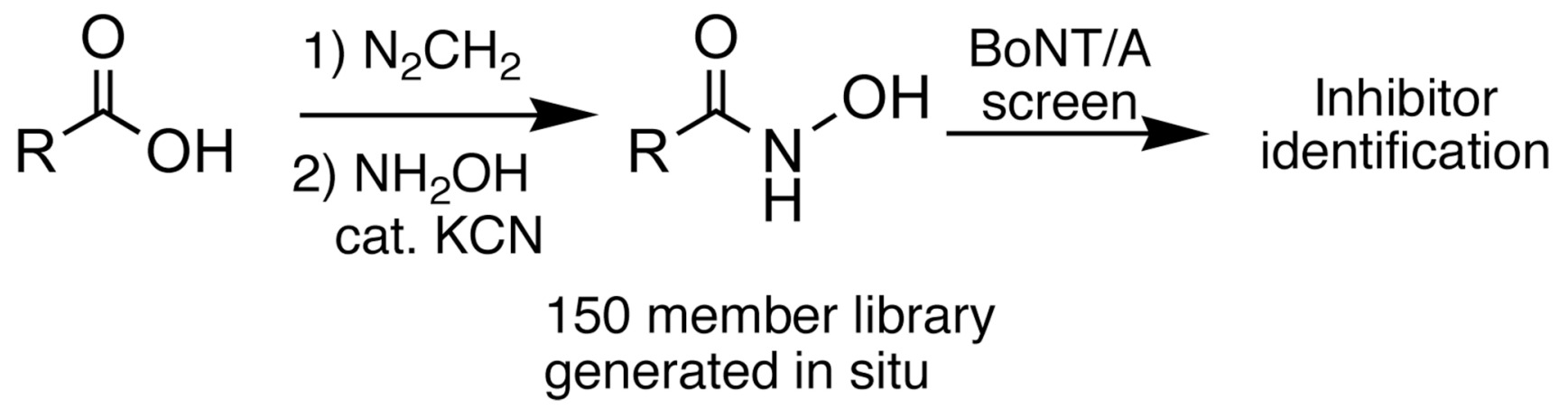

Scheme 1.

Synthesis and screening of in situ hydroxamate library. 


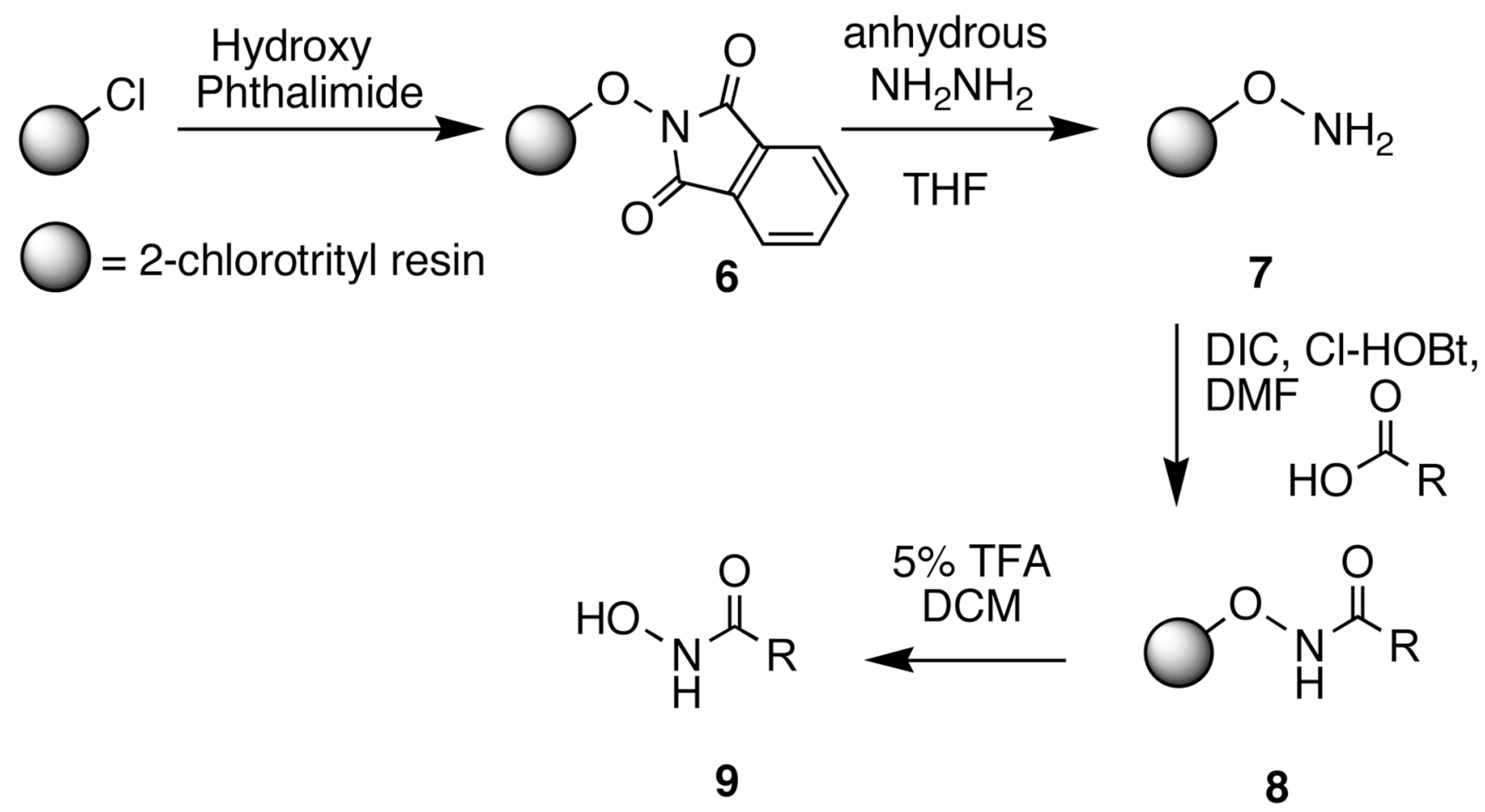

\section{Scheme 2.}

Solid-phase synthesis of hydroxamic acids 
Table 1

IC $_{50}$ values. $^{a}$

compound

structure

10

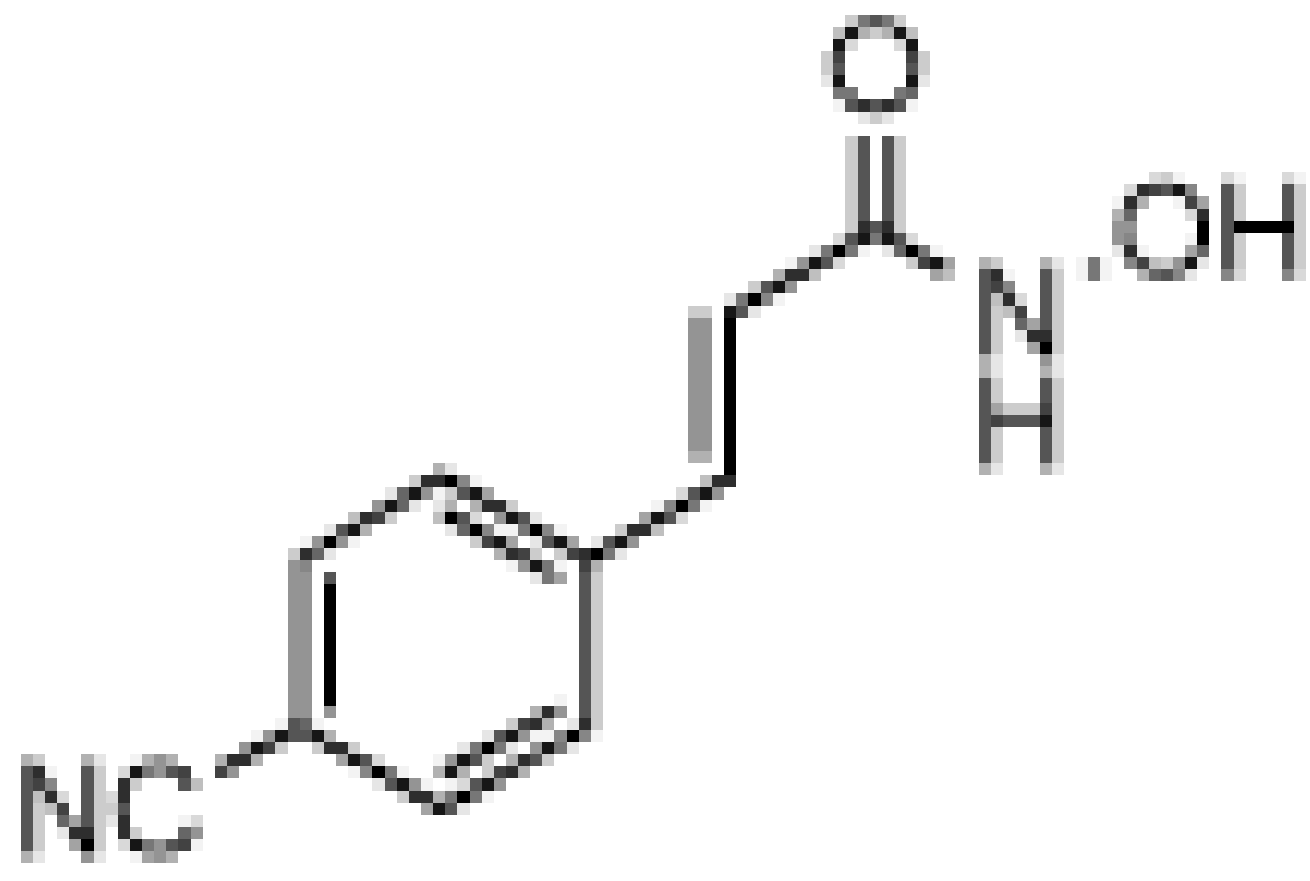

11

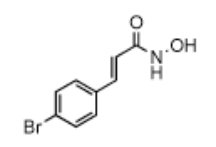

12

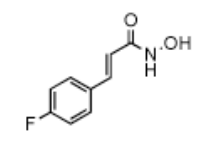

13

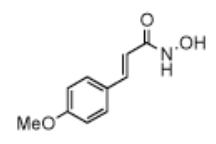

Org Lett. Author manuscript; available in PMC 2009 August 27. 


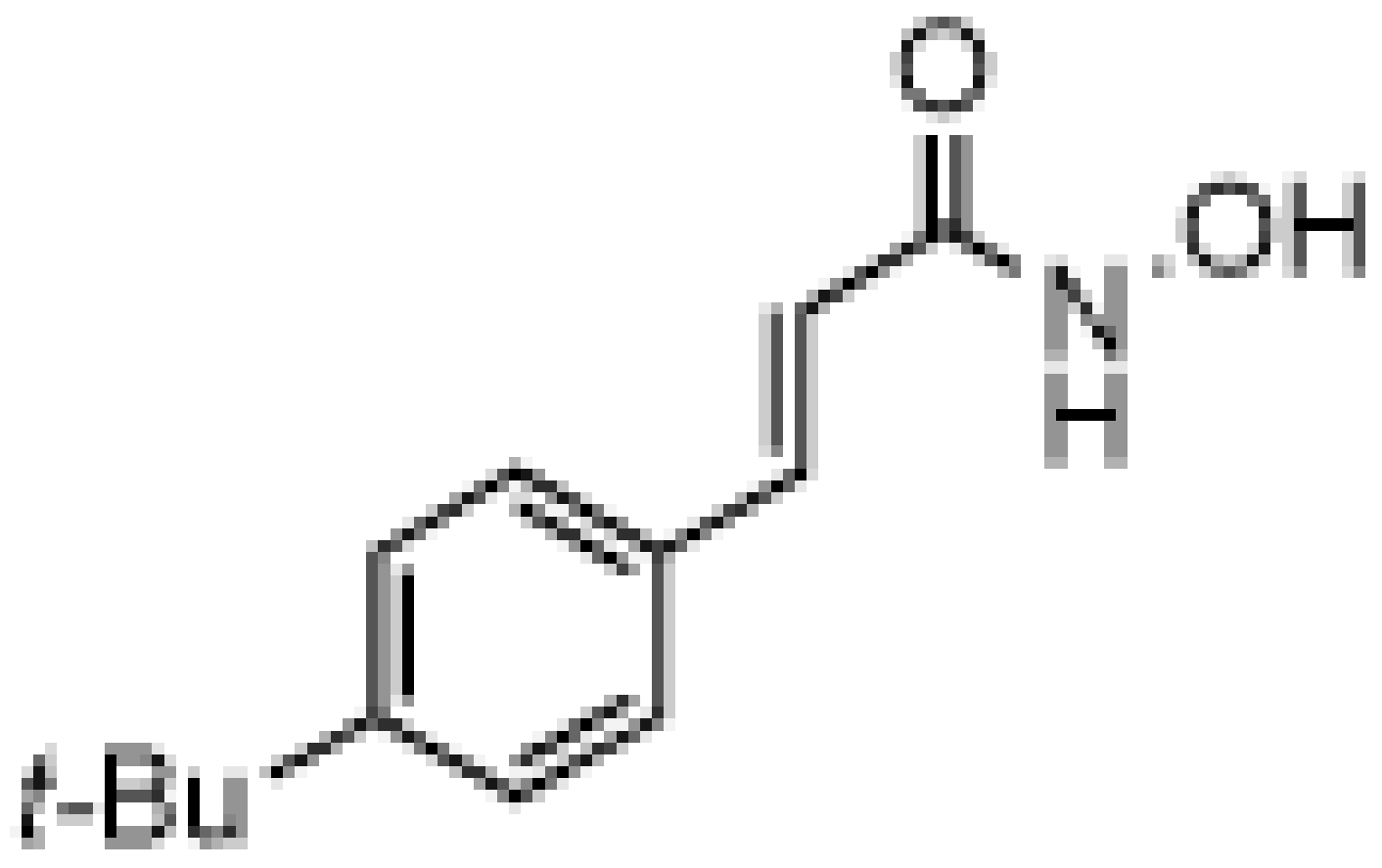

15

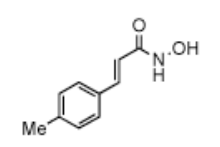

16

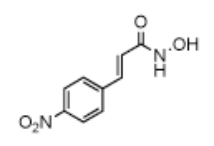

17

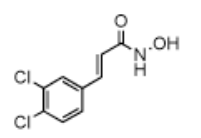

18

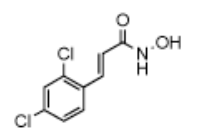



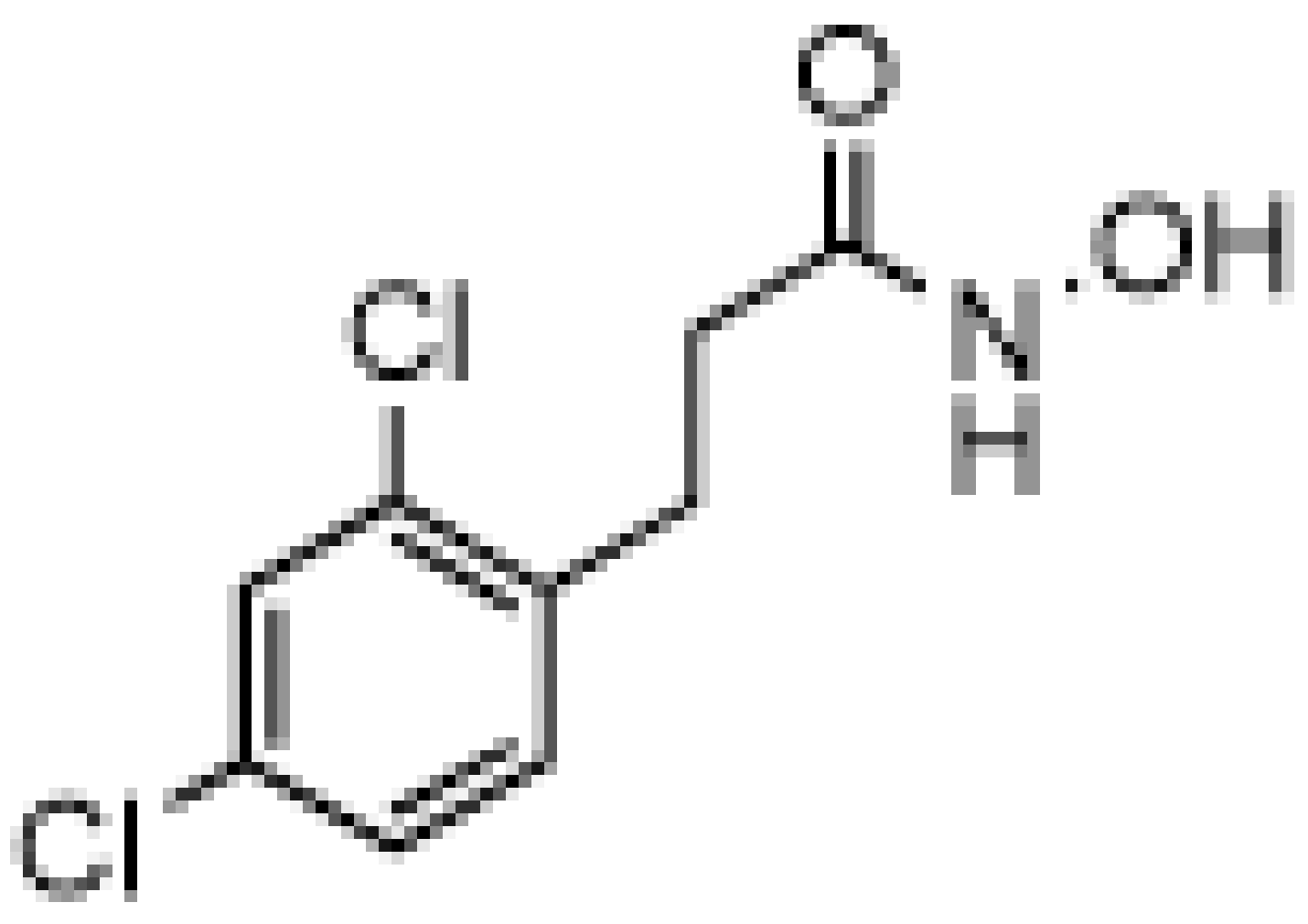

20

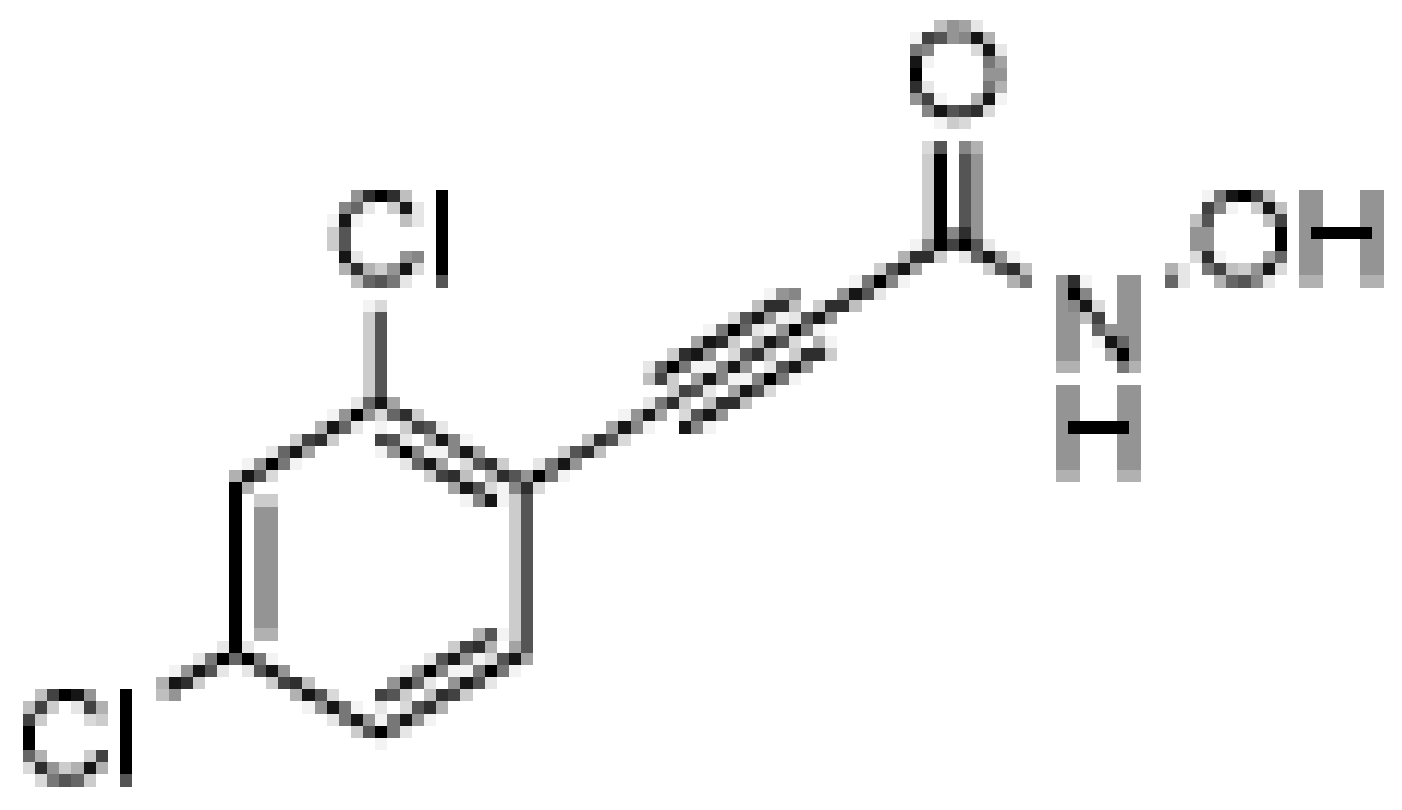

Org Lett. Author manuscript; available in PMC 2009 August 27. 
21 $\overbrace{F}$

${ }^{a}$ Assays were conducted at various inhibitor concentrations at $22.5^{\circ} \mathrm{C}, \mathrm{pH} 7.4$ in $40 \mathrm{mM}$ HEPES $0.01 \%$ (W/V) Tween® $20,5 \mu$ M SNAPtide substrate and $200 \mathrm{nM}$ enzyme. 